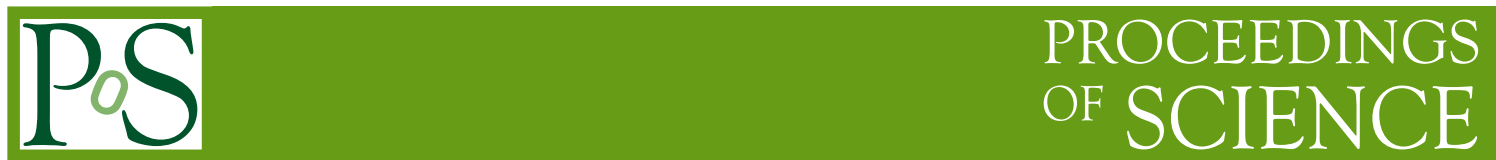

\title{
New Results from the Tevatron
}

\section{Costas VELLIDIS*广}

FNAL

E-mail: vellidis@fnal.gov

Recent results from the two Tevatron experiments, CDF and D0, in all areas of interest of highenergy physics are pesented. Most of the results are using the full integrated luminosity and contribute to the Tevatron legacy. In particular, the final Tevatron combinations of Higgs boson search limits, including all decay channels, and fits of Higgs boson couplings are shown. A significance of 3.0 standard deviations for the standard model Higgs boson is observed at the mass hypothesis of $125 \mathrm{GeV} / \mathrm{c}^{2}$. Relevant searches for new physics are also shown. Studies in depth of the forward-backward asymmetry in top-quark pair production show a persisting discrepancy with the standard model prediction at the level of two standard deviations. High-precision measurements of electroweak and chromodynamic processes place stringent constraints on standard model predictions. Measurements of very rare processes, mixing of states, and tiny asymmetries in exclusive heavy-flavored hadron production are testing fine details of the standard model.

XXI International Workshop on Deep-Inelastic Scattering and Related Subjects 22-26 April, 2013

Marseilles, France

\footnotetext{
${ }^{*}$ Speaker.

$\dagger$ On behalf of the CDF and D0 Collaborations.
} 


\section{Introduction}

The Tevatron proton-antiproton collider at the Fermi National Acceleraor Laboratory (Fermilab) in US was shut down on September 30, 2011, after nearly ten years of operations at a collision energy of $1.96 \mathrm{TeV}$ during Run II. In September 2011, dedicated runs at collision energies of 300 and $900 \mathrm{GeV}$ were conducted. The collider delivered $12 \mathrm{fb}^{-1}$ of integrated luminosity and each experiment, the Collider Detector at Fermilab (CDF) and D0, acquired approximately $10 \mathrm{fb}^{-1}$. About 15 billion collision events have been recorded by CDF and another 9 billion by D0. The corresponding data sets amount to about 10 and 9 petabytes, respectively, including Monte Carlo simulation samples. This sample is currently analyzed by the CDF and D0 Collaborations, formed by 60 and 70 member institutions from around the World, respectively. Each Collaboration involves currently about 400 active physicists from those institutions, working on the analysis of the data and publication of the results. The sample is well understood and, with the aid of the mature analysis knowledge developed by both Collaborations, it provides an enormous potential for physics within and beyond the standard model.

\section{Results}

Only a summary of the most relevant recent public results from the Tevatron experiments is reported here. All public results, with references to more detailed documentation, can be found in the public pages of the two experiments [1].

\subsection{Higgs}

Both Tevatron experiments have completed their program of searches for the standard model Higgs boson in all possible channels, using the full luminosity in almost all channels. The Tevatron combined results of the searches are submitted to Physical Review [2]. The left window of Figure 1 shows the exclusion limits of the combination at the 95\% confidence level (CL) as a function of the Higgs boson mass hypothesis. The dashed line shows the expected limit, with the one- and two-standard deviations uncertainty bands (green and yellow bands, respectively). The solid lind shows the observed limit. The Higgs boson is excluded between 150 and $180 \mathrm{GeV} / \mathrm{c}^{2}$, mainly by the $\mathrm{H} \rightarrow \mathrm{WW}$ channel, while there is an observed excess over the expectation between 115 and $150 \mathrm{GeV} / c^{2}$ with a maximum significance of 3.0 standard deviations at $125 \mathrm{GeV} / c^{2}$, mainly from the $\mathrm{H} \rightarrow \mathrm{b} \bar{b}$ channel. The dotted line shows the expectation for a Higgs boson with a mass of 125 $\mathrm{GeV} / c^{2}$. The observed line shows a clear preference for this hypothesis against the no Higgs boson hypothesis (dashed line).

The couplings of the standard model Higgs boson to the weak bosons and to the fermions wre derived from the measured cross sections times branching ratios in all production and decay channels. The right window of Figure 1 shows the measured fermionic and bosonic couplings with the one- and two-standard deviations uncertainty regions (green and yellow areas), compared to the standard model prediction. The sign degeneracy arises from the quadratic dependence of the cross sections and branching ratios on the couplings. The measurement is consistent with the prediction. 

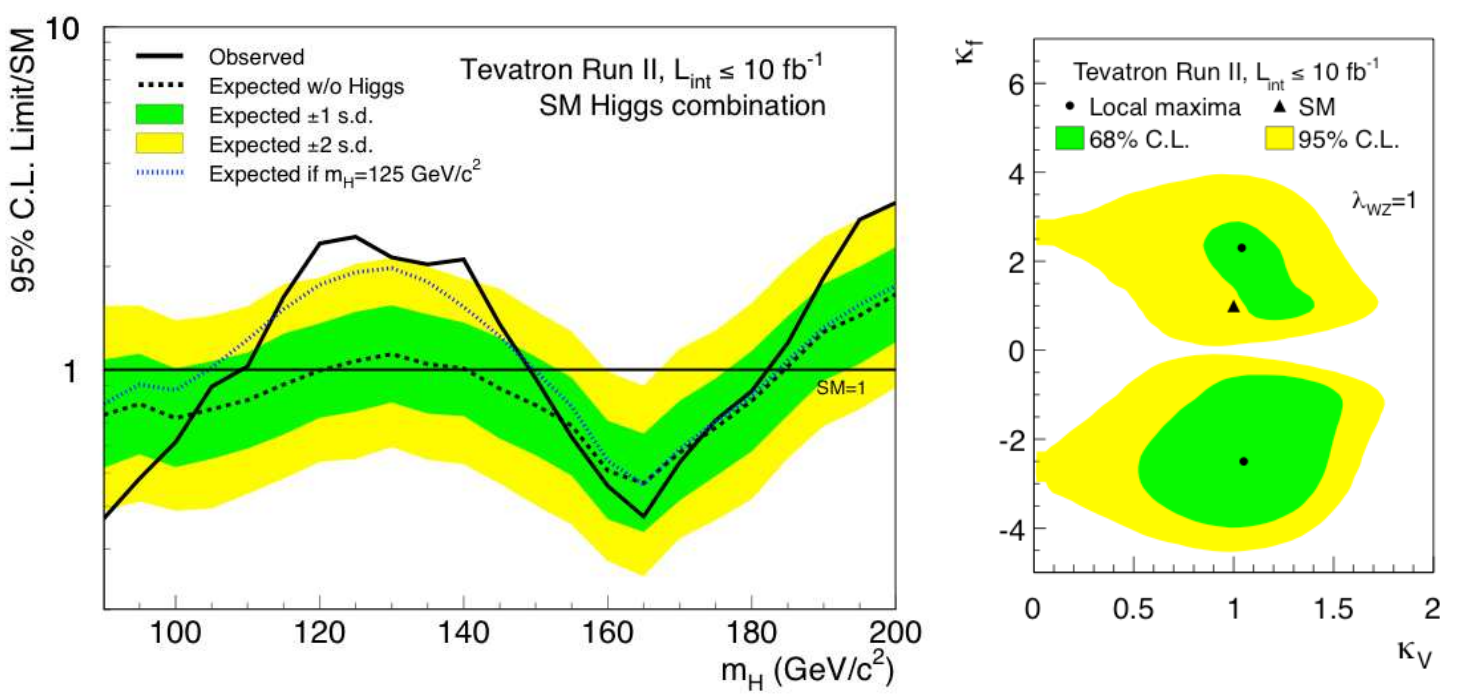

Figure 1: Exclusion limits of the standard model Higgs boson (left) and constraints on its couplings (right) from data corresponding to the full Tevatron luminosity in all production and decay channels.

\subsection{Exotics}

The left window of Figure 2 shows the expected exclusion limit from a search for $\mathrm{t} \overline{\mathrm{t}}$ resonances at $\mathrm{CDF}$ [3], as a function of the mass of the top-quark pair, compared with similar limits from LHC. The search at CDF is conducted in the $\bar{t} \rightarrow$ lepton+jets topology, using the full integrated luminosity of $9.4 \mathrm{fb}^{-1}$, requiring at least one b-tagged jet, and dividing the sample into three- and four or more-jets categories to optimize the sensitivity. The derived limit is currently the World's best up to a mass of $700 \mathrm{GeV} / \mathrm{c}^{2}$.
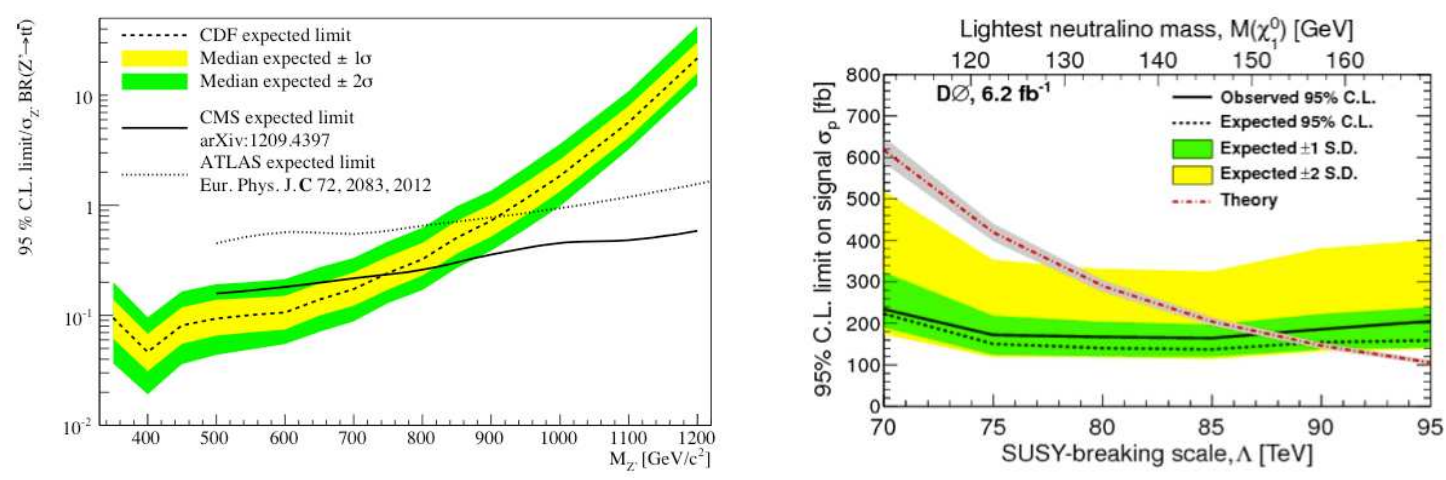

Figure 2: Exclusion limits of $Z^{\prime} \rightarrow t \bar{t}$ resonances as a function of the $Z^{\prime}$ mass (left) and of $Z \gamma+$ gravitino production as a function of both the supersymmetry breaking scale and the lightest neutralino mass (right).

The right window of Figure 2 shows the exclusion limits from a search for pair-produced neutralinos as next-to-lightest supersymmetric particles (NLSP) particles decaying into Z $\gamma+$ gravitino at D0 [4]. This signature is predicted in a model with gauge-mediated supersymmetry breaking (GMSB), with the gravitino in the role of the gaugino. The search is conducted by looking for a 
high-momentum photon plus a pair of high-momentum charged leptons (electrons or muons) plus high missing transverse energy using data corresponding to $6.2 \mathrm{fb}^{-1}$ of integrated luminosity. The model is excluded at $95 \% \mathrm{CL}$ up to a GMSB scale $\Lambda=87 \mathrm{TeV}$.

\subsection{Top quark}

The Tevatron experiments published last year, and updated early this year, a combination of the most precise measurements of the top-quark mass in the various decay channels [5], including measurements using the full data set. The combination yields a value of $\mathbf{M}_{\text {top }}=173.20 \pm 0.87$ $\mathrm{GeV} / c^{2}$, with a precision of $0.50 \%$. The left window of Figure 3 shows the breakdown of the uncertainties from the various measurements contributing to the combination. The right window shows $68 \%, 95 \%$, and $99 \%$ CL exclusion contours of the standard model Higgs boson in the plane of the $\mathrm{W}$ boson and top quark masses, compared with the World average measurements of these two masses and with allowed bands of the Higgs boson mass from direct searches at the LHC. There is a remarkable consistency between the measured values of the top quark and the $\mathrm{W}$ and Higgs bosons, consolidating the consistency of the standard model.
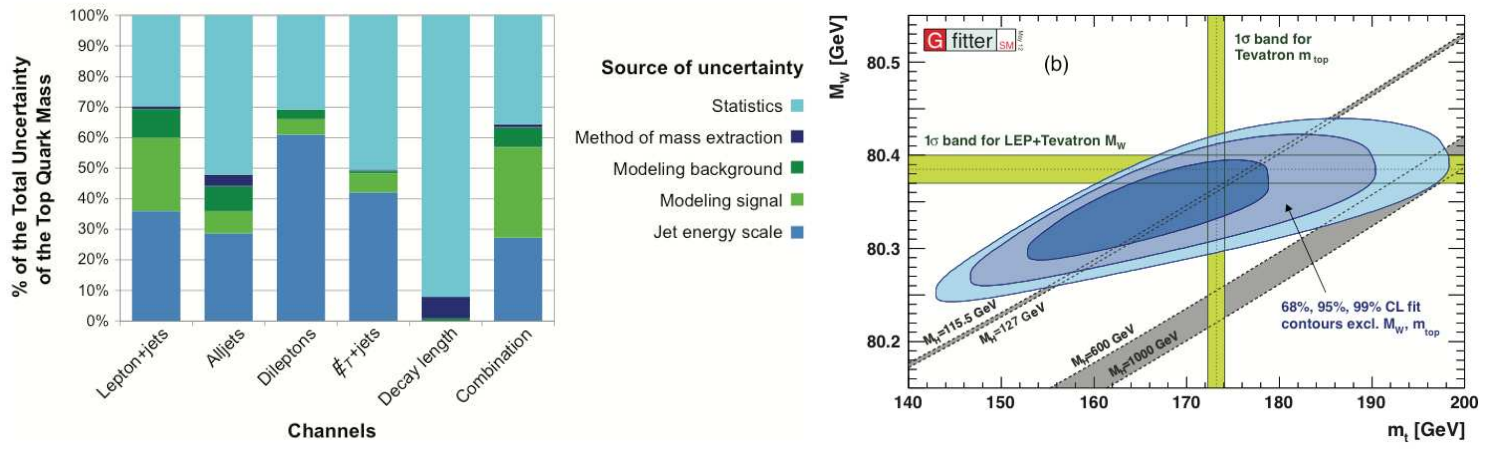

Figure 3: Contribution of uncertainties per measurement in the top-quark mass Tevatron combination (left) and constraints on the standard model Higgs boson mass from the World average of the top-quark and Wboson masses (right).

CDF recently measured the cross section for $t \bar{t}$ production in the lepton + jets decay channel, differential in the cosine of the top-quark production angle about the proton direction in the $t \bar{t}$ rest frame, using the full intergated luminosity of $9.4 \mathrm{fb}^{-1}$ [6]. The cross section was analyzed in terms of Legende polynomials, to determine the moments characterizing the shape of the distribution. This shape is directly related with the forward-backward $t \bar{t}$ production asymmetry $A_{F B}$ in the lab frame and thus the moments provide an insight to the asymmetry. The left window of Figure 4 shows the contribution of first few moments to $A_{F B}$ for the data and for various calculations predicting a non-vanishing asymmetry. An agreement with the standard model calculation at nextto-leading order (NLO) with respect to the strong and electroweak couplings is observed for all but the first moment. It is seen that $\mathrm{A}_{\mathrm{FB}}$ is dominated by an anomalously large first moment.

\subsection{Electroweak interactions}

D0 searched for anomalous WWZ and WW $\gamma$ trilinear gauge couplings (aTGC) [7]. The search was conducted using $\mathrm{WW}+\mathrm{WZ} \rightarrow 1 v \mathrm{jj}, \mathrm{WW} \rightarrow 1 v \mathrm{l}, \mathrm{WZ} \rightarrow 1 v 1 \mathrm{l}$, and $\mathrm{W} \gamma \rightarrow 1 v \gamma$ samples for various 
integrated luminosities, assuming a new physics scale of $\Lambda=2 \mathrm{TeV}$. The right window of Figure 4 shows $68 \%$ and $95 \%$ CL contour plots of the aTGC fit compared with the standard model prediction. The fit is consistent with the prediction.
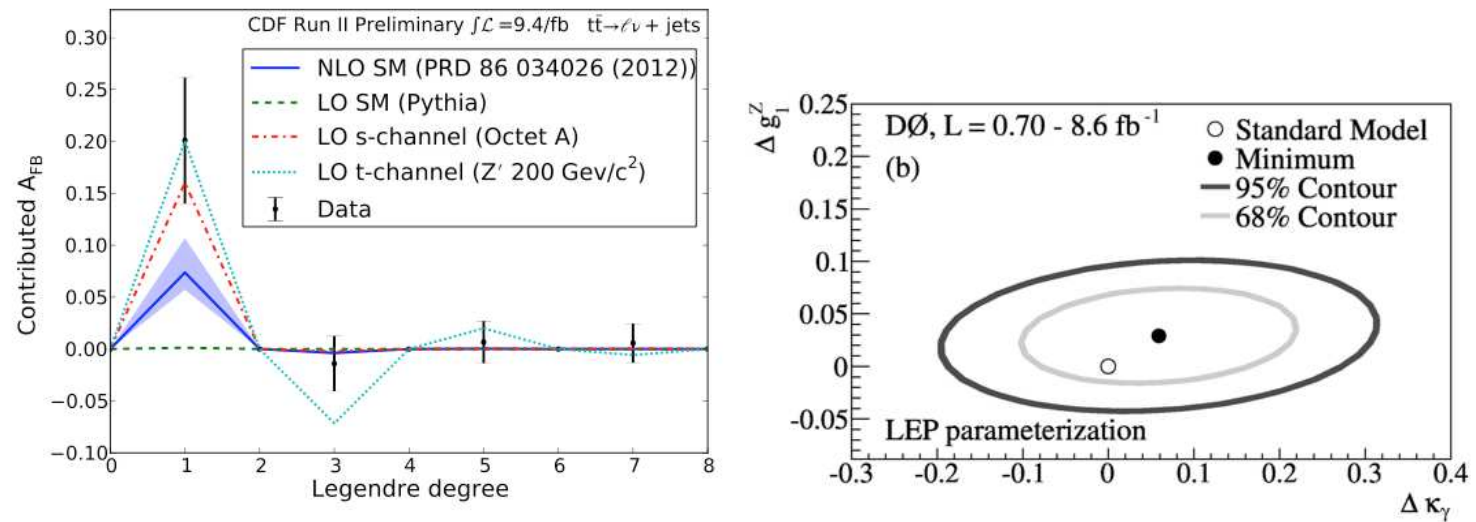

Figure 4: Contribution of Legendre moments of the $t \bar{t}$ angular distribution to the forward-backward $t \bar{t}$ production asymmetry (left) and constraints on the anomalous trilinear gauge couplings (right).

CDF extracted the weak angle from a measurement of the V-A interference term $\left(\mathrm{A}_{4}\right)$ of the angular distribution of $\mathrm{e}^{+} \mathrm{e}^{-}$decays in the mass range between 66 and $116 \mathrm{GeV} / c^{2}$ [8] using data corresponding to $2.1 \mathrm{fb}^{-1}$ of integrated luminosity. The effective sine-squared of the weak angle $\sin \theta_{\text {eff }}^{\text {lep }}=0.2309 \pm 0.0010$, corrected for radiative effects at the leptonic decay vertex, was derived from the measured $\mathrm{A}_{4}$ using a soft gluon-resummed standard model calculation. The same measurement was used to extract a value of the $\mathrm{W}$ boson mass $\mathrm{M}_{W}=80.297 \pm 0.055 \mathrm{GeV} / 2$. The left window of Figure 5 shows the comparison of the extracted $\sin \theta_{\mathrm{eff}}^{\text {lep }}$ with other extractions from LEP and D0. The right window of Figure 5 shows the comparison of the indirect measurement of the $\mathrm{W}$ boson mass with recent and older World average direct measurements.

\subsection{QCD}

CDF studied the underlying event in hard dijet production using the full data sets at the the three collision energies of 300, 900, and $1960 \mathrm{GeV}$ [9]. The study is done by measuring distributions of particles emitted normally to the direction of the two jets, which emerge from the underlying event. The left window of Figure 6 shows the distribution of scalar sums of the transverse momenta of charged particles emitted in the normal direction, in comparison with out-of-the-box predictions of the PYTHIA "tune Z1" Monte Carlo event generator for the three collision energies. A reasonable agreement between the data and the predictions is observed.

$\mathrm{D} 0$ recently measured the cross section for $\mathrm{W}$ boson production associated with jets, differential in various kinematic variables and for various jet multiplicities, using data corresponding to 3.7 $\mathrm{fb}^{-1}$ of integrated luminosity [10]. The right window of Figure 6 shows the cross section differential in the azimuthal angle between the two leading jets in the event, compared with predictions from QCD-based state-of-the-art calculations. A resonable overall agreement between the data and the NLO calculations is observed. 

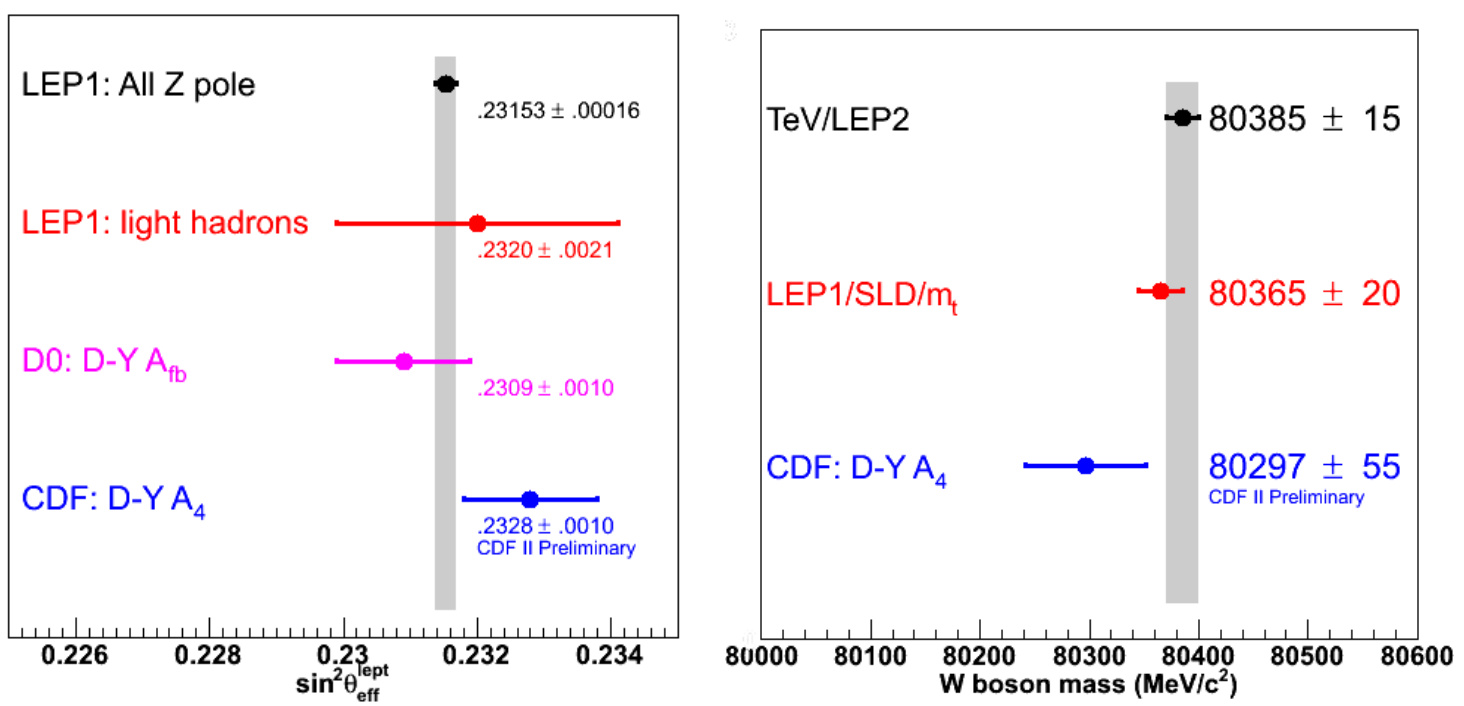

Figure 5: The effective sine-squared of the weak angle (left) and the indirect measurement of the W boson mass (right) derived from the angular distribution of Drell-Yan $\mathrm{e}^{+} \mathrm{e}^{-}$pairs and compared with other measurements.

\subsection{Heavy flavor}

The Tevatron sample has the advantage of a CP-invariant initial state and, therefore, it is ideal for measurements of tiny $\mathrm{CP}$ violation asymmetries $\left(\mathrm{A}_{\mathrm{CP}}\right)$ which test fine details of the standard model and probe for new physics. CDF conducted a set of measurements in exclusive charmless bottom decays into two charged hadrons, looking for $\mathrm{A}_{\mathrm{CP}}$ in $\mathrm{B}$ meson and $\Lambda_{\mathrm{b}}$ baryon decays [11]. The left window of Figure 7 shows the mass distributions of charged pion pairs from which the $\mathrm{B}^{0}$ meson is reconstructed. The results are consistent with corresponding results from b-factories and from the LHCb. In particular, an $A_{C P}$ is observed for with a significance of 5 standard deviations the $\mathrm{B}^{0}$ meson and with a significance of 2.9 standard deviations for the $\mathrm{B}^{0}{ }_{\mathrm{s}}$ meson, which raises to 4.5 standard deviations when combined with the LHCb result.

A similar measurement was recently conducted by D0 [12], looking for $\mathrm{A}_{\mathrm{CP}}$ in $\mathrm{B}^{ \pm} \rightarrow \mathrm{J} \psi \mathrm{K}^{ \pm} \rightarrow$ $\mu^{+} \mu^{-} \mathrm{K}^{ \pm}$and $\mathrm{B}^{ \pm} \rightarrow \mathrm{J} \psi \pi^{ \pm} \rightarrow \mu^{+} \mu^{-} \pi^{ \pm}$decays. The right window of Figure 7 shows the mass distribution of the muon-pair plus kaon or pion system, from which the charged B meson is reconstructed. $\mathrm{An}_{\mathrm{CP}}$ of $(0.59 \pm 0.36) \%$ for the $\mathrm{J} \psi \mathrm{K}$ system and of $(-4.2 \pm 4.8) \%$ for the $\mathrm{J} \psi \pi$ system is obtained. Despite the large uncertainties, these results are the most accurate $\mathrm{A}_{\mathrm{CP}}$ measurements in leptonic channels to-date, thanks to the ability of the D0 detector to regularly reverse the magnetic field of the spectrometer, which cancels detector asymmetries.

\section{Future}

There is an important physics program yet to complete with the Tevatron sample. Priority is given to measurements relevant to the unique Tevatron energy, initial state, and collision environment. The program includes combinations of results from CDF and D0, to achieve maximum precision, and, when appropriate, also from Tevatron and LHC. In the domain of searches for new 

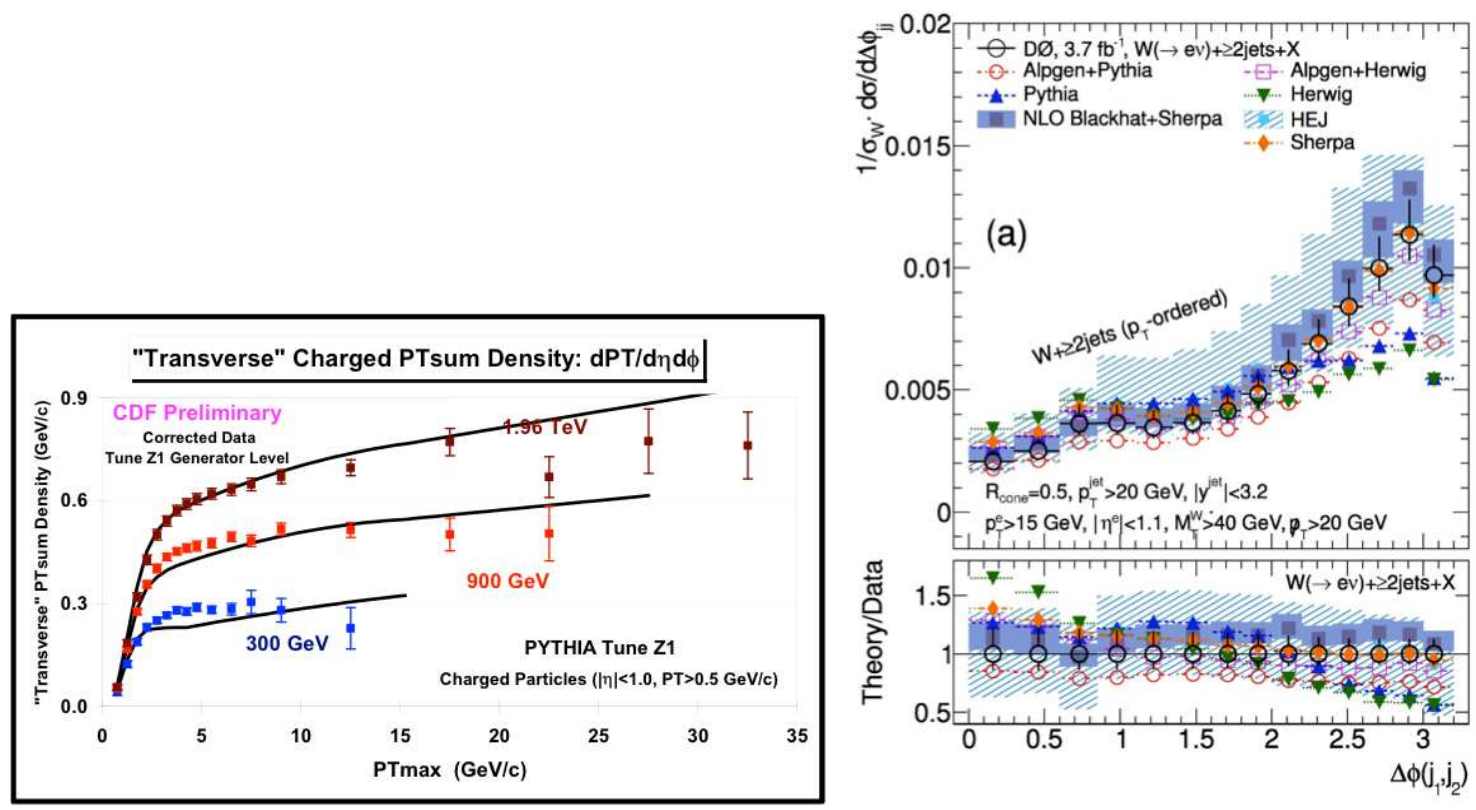

Figure 6: Distribution of sums of charged particle transverse momenta at three collision energies (left) and differential cross section for $\mathrm{W}+$ jets production as a function of the azimuthal angle between the two leading jets in the event (right).

physics, the focus is on searches where trigger thresholds and backgrounds favor Tevatron against LHC regarding better low-mass lever arm and optimal sensitivity, respectively. For the standard model, the plan is to exhaust precision capacity of legacy measurements such as the masses of the $\mathrm{W}$ boson and of the top quark, the forward-backward asymmetry in top-quark pair production, and the s-channel single top production. In the field of heavy flavor physics, the goal is to fully exploit the advantage of the Tevatron CP-invariant initial state in order to measure observables relevant to $\mathrm{CP}$ violation and to study heavy flavor production properties.

\section{Conclusions}

The Tevatron experiments, CDF and D0, keep producing high-quality physics results. The standard model Higgs boson search program is now complete, providing an independent evidence for the existence of this particle, complementary to its recent observation by the LHC experiments. High-precision measurements of the top quark and the $\mathrm{W}$ boson masses show consistency, within the standard model, with the mass of the Higgs boson measured at the LHC. Stringent tests of the standard model are performed in the areas of the top quark, of electroweak interactions, of chromodynamic processes, and of heavy-flavored bound states. Constraints on new physics models are placed by direct searches for new paricles. A joint effort by the Fermilab Computing Sector and the $\mathrm{CDF}$ and $\mathrm{D} 0$ Collaborations is ongoing, aimed to preserve the data and the analysis knowledge of the unique Tevatron proton-antiproton sample. 

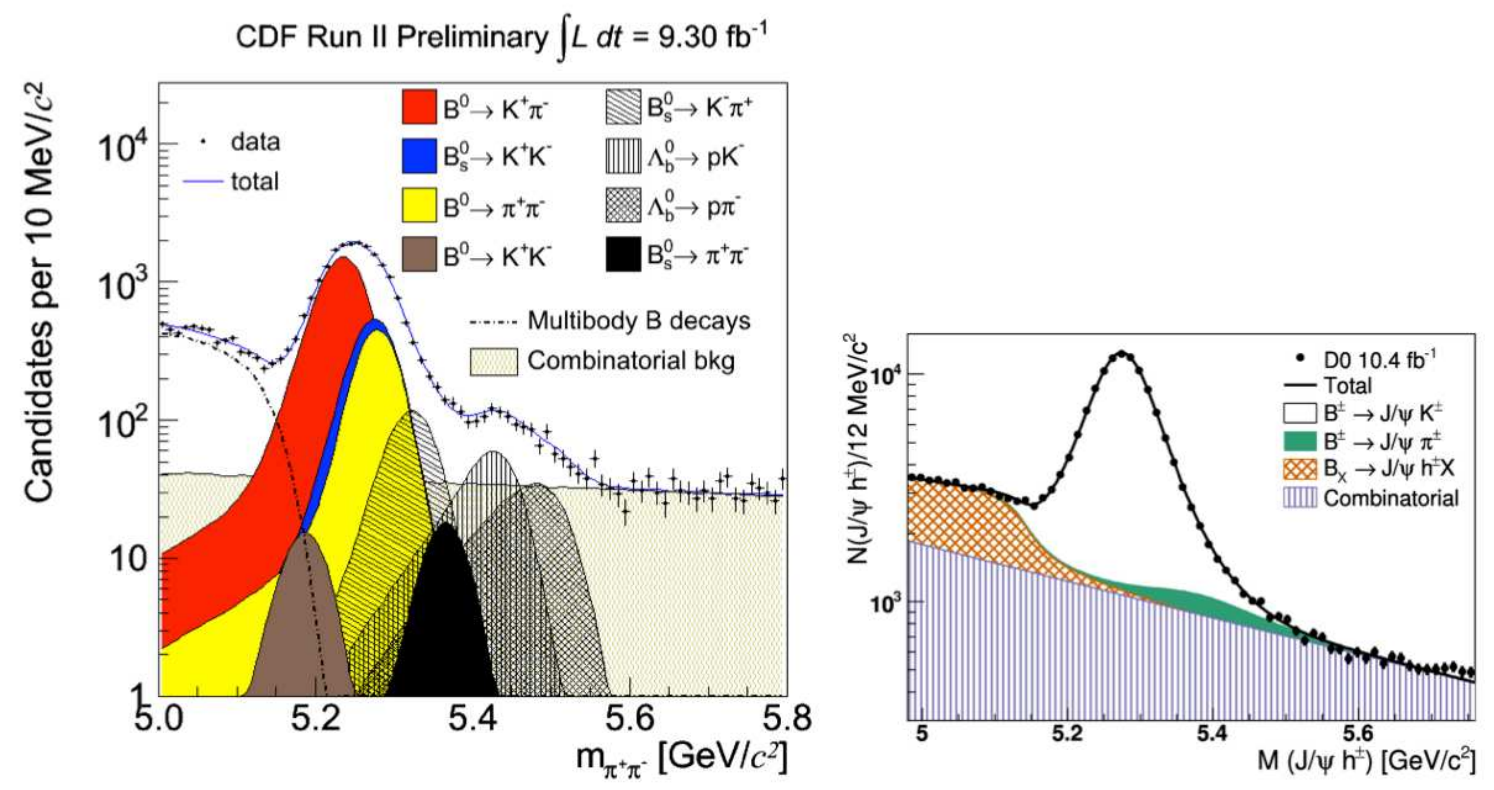

Figure 7: Mass distributions of charmless $\mathrm{B} \rightarrow \mathrm{hh}$ decays (left) and of $\mathrm{B} \rightarrow \mu \mu \mathrm{h}$ decays (right).

\section{References}

[1] www-cdf.fnal.gov/physics/physics.html; www-d0.fnal.gov/Run2Physics/WWW/results.html

[2] T. Aaltonen et al. (CDF and D0 Collaborations), arXiv:hep-ex/1303.6346, submitted to Phys. Rev. D.

[3] T. Aaltonen et al. (CDF Collaboration), Phys. Rev. Lett. 110, 121802 (2013).

[4] V. M. Abazov et al. (D0 Collaboration), Phys. Rev. D 86, 071701(R) (2012).

[5] T. Aaltonen et al. (CDF and D0 Collaborations), Phys. Rev. D 82, 096003 (2012); CDF public note 10976.

[6] T. Aaltonen et al. (CDF Collaboration), CDF public note 10974.

[7] V. M. Abazov et al. (D0 Collaboration), Phys. Lett. B 718, 451 (2012).

[8] T. Aaltonen et al. (CDF Collaboration), Phys. Rev. Lett. 106, 241801 (2011); CDF public note 10952.

[9] T. Aaltonen et al. (CDF Collaboration), CDF public note 10874.

[10] V. M. Abazov et al. (D0 Collaboration), arXiv:hep-ex/1302.4508, submitted to Phys. Rev. D.

[11] T. Aaltonen et al. (CDF Collaboration), CDF public note 10726.

[12] V. M. Abazov et al. (D0 Collaboration), arXiv:hep-ex/1304.1655, accepted in Phys. Rev. Lett. 